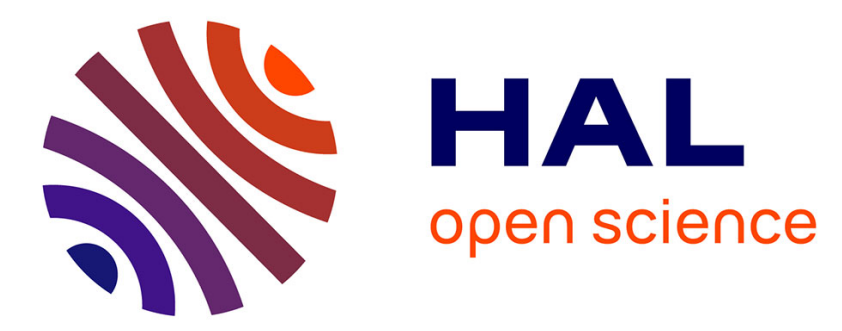

\title{
Control design for an automotive turbine rankine cycle system based on nonlinear state estimation
}

Johan Peralez, Madiha Nadri, Pascal Dufour, Paolino Tona, A Sciarretta

\section{To cite this version:}

Johan Peralez, Madiha Nadri, Pascal Dufour, Paolino Tona, A Sciarretta. Control design for an automotive turbine rankine cycle system based on nonlinear state estimation. 53rd IEEE Conference on Decision and Control (CDC), Dec 2014, Los Angeles, CA, United States. pp.3316-3321, 10.1109/CDC.2014.7039902 . hal-01265062

\section{HAL Id: hal-01265062 \\ https://hal.science/hal-01265062}

Submitted on 1 Feb 2016

HAL is a multi-disciplinary open access archive for the deposit and dissemination of scientific research documents, whether they are published or not. The documents may come from teaching and research institutions in France or abroad, or from public or private research centers.
L'archive ouverte pluridisciplinaire HAL, est destinée au dépôt et à la diffusion de documents scientifiques de niveau recherche, publiés ou non, émanant des établissements d'enseignement et de recherche français ou étrangers, des laboratoires publics ou privés. 
This document must be cited according to its final version which is published in a conference as:

J. Peralez, M. Nadri, P. Dufour, P. Tona, A. Sciarretta, "Control design for an automotive turbine rankine cycle system based on nonlinear state estimation", 53rd IEEE Conference on Decision and Control (CDC), Los Angeles, CA, USA,

pp. 3316-3321, december 15-17, 2014.

DOI : $10.1109 /$ CDC.2014.7039902

You downloaded this document from the CNRS open archives server, on the webpages of Pascal Dufour: http://hal.archives-ouvertes.fr/DUFOUR-PASCAL-C-3926-2008 


\title{
Control Design for an Automotive Turbine Rankine Cycle System based on Nonlinear State Estimation
}

\author{
Johan Peralez, Madiha Nadri, Pascal Dufour, Paolino Tona, Antonio Sciarretta
}

\begin{abstract}
This paper investigates the problem of control design for an Organic Rankine Cycle (ORC) for Waste Heat Recovering (WHR). Based on a moving boundary heat exchanger model derived from energy and mass conservation principles, an implicit model, control-oriented and experimentally validated, is presented. Then, a first controller which consists on a gain-scheduled PID combined to a dynamic feedforward is implemented on the pump speed to maintain the superheat close to the set point value. A second controller is a nonlinear feedback law which allows to adjust the evaporating pressure to time-varying demand with a good accuracy. To implement these two controllers, an observer is designed to achieve the state estimation from pressure measurement, typically available in WHR applications.

The observer and the control approach are validated on realistic operating conditions.
\end{abstract}

\section{INTRODUCTION}

In recent years, growing awareness of environmental and energy issues has steadily driven research interest in technologies enabling efficiency improvements for internal combustion engines. Engine waste heat recovery, in particular, has a interesting potential to increase fuel thermal efficiency, reduce fuel consumption, and decrease engine emissions. Indeed, more than one third of the energy produced by internal combustion engines is wasted through exhaust gas heat. The ORC is a thermodynamical cycle that seeks to recover the waste heat by producing expansion work. A pump circulates an organic working fluid in a closed loop where an external heat source supplies heat, via a heat exchanger. Vaporized fluid expands in a turbine or an expander to produce mechanical power. Among all the currently available technical solutions used to recover low-grade waste heat, the ORC combines the benefits of a high thermal efficiency and of a limited impact on engine operation, and has thus drawn the attention of many car and truck manufacturers [1].

In transport applications, the main difference with conventional (stationary) ORCs lies in the highly transient behavior of the hot source, depending on driving conditions. This poses a great challenge for system

J. Peralez, P. Tona and A. Sciarretta are with the Control, Signal and System Department at IFP Energies Nouvelles, France \{johan.peralez, paolino.tona, antonio.sciarretta\}@ifpen.fr

P. Dufour and M. Nadri are with the Université de Lyon, F-69622, Lyon, France - Université Lyon 1, Villeurbanne, France - CNRS, UMR 5007, LAGEP, France \{dufour, nadri\}@lagep. univ-lyon1.fr control. In this context, an effective control system is essential to attain satisfactory performance over a broad range of operating conditions, acting as time-varying disturbances. This is especially true when there are few available actuators and sensors, as it often happens in the automotive industry. Despite that, literature on control design for these applications is still very scarce and most strategies for vapour pressure and temperature control are based on linear models [2]-[4].

The system considered here is illustrated in Fig. 1. It uses a turbine connected to an electric generator which delivers power $\left(P_{L}\right)$ to the on-board electrical system. A by-pass valve controls the fraction of exhaust gas entering the evaporator. Despite the importance of this component, which is mandatory for safety reasons, only heuristic control approaches have been reported [5]-[7].

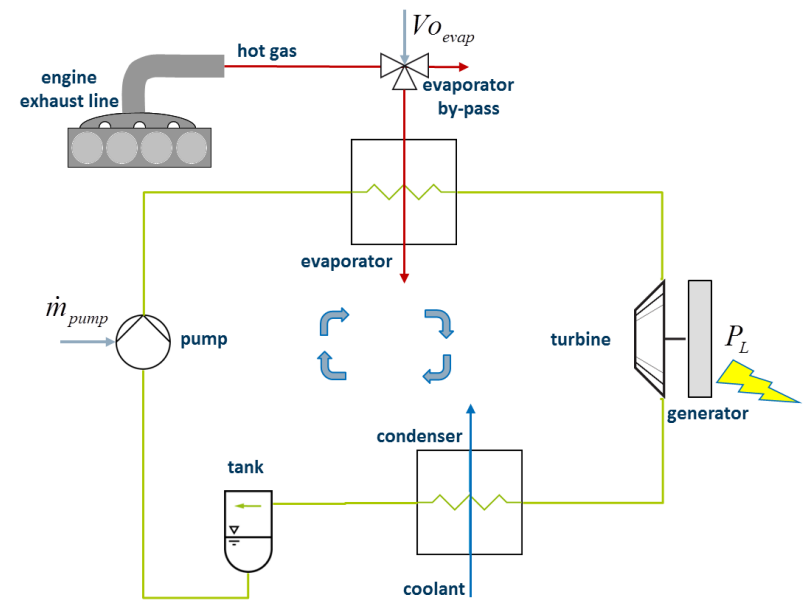

Fig. 1. The Organic Rankine Cycle system under investigation

In a previous paper [7], we have shown - with an experimental validation - that a nonlinear model-based monovariable control approach is superior to the commonly used PID approach for superheat regulation. In the present paper, we extend the approach to the multivariable case, with the use of the evaporator by-pass as an additional actuator, allowing tracking a pressure setpoint - or equivalently of a power production demand.

The paper is organized as follows. In the first section we briefly introduce the reference and the simplified models. Then the control problem is formulated. In the third section, a new multivariable control scheme is introduced. In section 4, an implicit observer is designed which allows state estimation using the pressure measurement. 
The complete approach is illustrated in the last section.

\section{Model AND PROBlem FORMUlation}

Heat exchangers are the main contributors to thermal inertia and therefore are the focus of attention in dynamic modelling.

\section{A. Partial Differential Equation (PDE) reference model}

A classic 1-D representation of heat exchangers (along the fluid displacement direction $z$ ) is given by mass and energy balances for the fluid and the wall [8]. The evolution of fluid and wall variables - namely fluid mass flow $\dot{m}$, specific enthalpy $h$, pressure $p$ and wall temperature $T_{w}$ - is then described by

$$
\left\{\begin{array}{c}
\frac{\partial(\rho A)}{\partial t}+\frac{\partial \dot{m}}{\partial z}=0 \\
\frac{\partial(\rho A h-A p)}{\partial t}+\frac{\partial \dot{m} h}{\partial z}=\pi D \alpha\left(T_{w}-T\right) \\
C_{w} \rho_{w} A_{w} \frac{\partial T_{w}}{\partial t}=\pi D \alpha\left(T-T_{w}\right)+\pi D_{o} \alpha_{o}\left(T_{a}-T_{w}\right)
\end{array}\right.
$$

where $A, D, D_{o}, \rho_{w}, C_{w}$ are design parameters. Density $\rho$ and temperature $T$ of the fluid are nonlinear maps of $p$ and $h . T_{a}$ is the ambient temperature. $\alpha$ (resp. $\alpha_{o}$ ) is the heat exchange coefficient between the wall and the working fluid (resp. the ambient).

Notice that the above model is a system of three coupled nonlinear PDEs. Such a representation is difficult to deal with for control purposes. Therefore, a discretization of system (1) is used in simulation for control validation. Among the methods for discretization of system (1), the use of moving boundaries (MB) results in a relatively low order model. As shown in Fig. 2, the MB model monitors the length of each fluid phase along the evaporator: the normalized zone lengths $L_{1}, L_{2}, L_{3}$ track the liquid, two-phase and vapour zones, respectively. Fluid and wall dynamics are then captured by seven state variables: $x=\left[\begin{array}{lllllll}T_{w 1} & T_{w 2} & T_{w 3} & L_{1} & L_{2} & h_{3} & p\end{array}\right]^{T}$. A more detailed representation on this model may be found in $[8]$ and is referred as the reference model in the following.

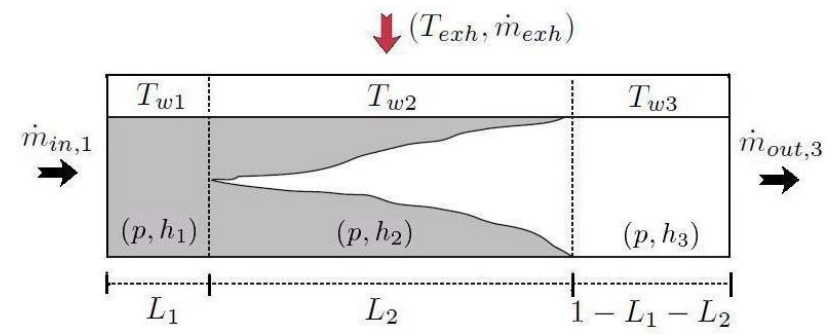

Fig. 2. Moving boundaries layout for the evaporator.

\section{B. Control-oriented simplified model}

In literature, model-based control generally circumvents the complexity of a reference model, such the one described above, by linearization around an operating point. In [7], we proposed a simplified model, experimentally validated over a broad range of time varying operating conditions. To achieve this, we assume a twotime-scale dynamic behaviour where wall temperatures capture the slow dynamics. Moreover, it was shown that the high and low pressure parts of the ORC could be decoupled, resulting in the simplified model described in the following.

Wall energy balance and fluid equilibrium yield the following equations for each of the three zones:

$$
\begin{gathered}
\frac{d T_{w i}}{d t}=-\beta_{i}\left[T_{w i}(t)-T_{i}(p(t), S H(t))\right] \\
-\eta_{e x h}(t)\left[T_{w i}(t)-T_{e x h}(t)\right], \\
\left\{\begin{array}{l}
L_{i}(t)=\dot{m} \frac{\Delta h_{i}(p(t), S H(t))}{\beta_{i}\left(T_{w i}(t)-T_{i}(p(t), S H(t))\right)} \\
\sum_{i=1}^{3} L_{i}(t)=1,
\end{array}\right.
\end{gathered}
$$

where $S H$ is the superheating value, the $\beta_{i}$ s are the components of a constant vector, $T_{i}(p(t), S H(t))$ and $\Delta h_{i}(p(t), S H(t))$ are nonlinear maps of fluid temperature and enthalpy and:

$$
\eta_{e x h}(t)=\dot{m}_{e x h}(t) \beta_{5}\left[1-\exp \left(-\frac{\beta_{6}}{V_{o}(t) \dot{m}_{e x h}(t)}\right)\right] .
$$

The heat exchanger model is completed by a static model of the turbine mass flow:

$$
\dot{m}(t)=\beta_{4} \sqrt{\rho(p(t), S H(t)) p(t)},
$$

while a nonlinear map for power production $P_{L}(p(t), S H(t))$ is considered.

Looking at expressions (2) and (3), it can be seen that the obtained control-oriented model is a nonlinear implicit model.

\section{Problem formulation}

The control problem is to respond to a power production demand while ensuring a safe level for pressure and superheating. From the map of power production, power demand can be converted in term of pressure set-point. So let $y=\left[\begin{array}{ll}S H & p\end{array}\right]^{T}, T_{w}=\left[\begin{array}{lll}T_{w 1} & T_{w 2} & T_{w 3}\end{array}\right]^{T}$ and $u=\left[\begin{array}{ll}\dot{m} & \eta_{\text {exh }}\end{array}\right]^{T}$.

This two-input two-output control problem is treated in two steps, assuming a two-time scale dynamic behavior. First, the control design proposed in [7] is used to regulate the superheating $y_{1}$ with pump mass flow $u_{1}$. Indeed, experimental results in [7] showed that a control based on a gain-scheduled PID with a dynamic feedforward allows a tight superheating regulation. Then, the control design for the pressure $y_{2}$ assumes the (fast) superheating dynamic as perfectly regulated to tune the evaporator by-pass $u_{2}$. Furthermore, this control requires knowledge of all system states. To overcome this problem, the authors in [7] used an open-loop model for the estimation of needed states. 
In this work, we propose to use an observer to improve the robustness of the closed loop against the model uncertainties. Then, a second nonlinear controller for pressure will be designed. The control scheme proposed in [7] is taken up and completed as shown in Fig. 3.

Assuming that the pump mass flow is fixed by the value of $S H$ following (5), the system of equations (3)-(5) can be rewritten in the following semi-explicit form

$$
\left\{\begin{array}{c}
\dot{T_{w}}=f\left(T_{w}, p\right)+u_{2} g\left(T_{w}, t\right) \\
\varphi\left(T_{w}, p\right)=0
\end{array}\right.
$$

where

$$
\begin{array}{r}
f\left(T_{w}, p\right)=\left(\begin{array}{l}
\beta_{1}\left(T_{1}(p)-T_{w 1}\right) \\
\beta_{2}\left(T_{2}(p)-T_{w 2}\right) \\
\beta_{3}\left(T_{3}(p)-T_{w 3}\right)
\end{array}\right), \\
g\left(T_{w}, t\right)=\left(\begin{array}{l}
T_{e x h}(t)-T_{w 1} \\
T_{e x h}(t)-T_{w 2} \\
T_{e x h}(t)-T_{w 3}
\end{array}\right), \\
\varphi\left(T_{w}, p\right)=1-\left(L_{1}+L_{2}+L_{3}\right),
\end{array}
$$

with

$$
\begin{aligned}
L_{1} & =u_{1} \frac{h_{l}(p)-h_{i n}}{\beta_{1}\left(T_{w 1}-T_{1}(p)\right)}, \\
L_{2} & =u_{1} \frac{h_{v}(p)-h_{l}(p)}{\beta_{2}\left(T_{w 2}-T_{2}(p)\right)} \\
L_{3} & =u_{1} \frac{h_{\text {out }}(p)-h_{v}(p)}{\beta_{3}\left(T_{w 3}-T_{3}(p)\right)}
\end{aligned}
$$

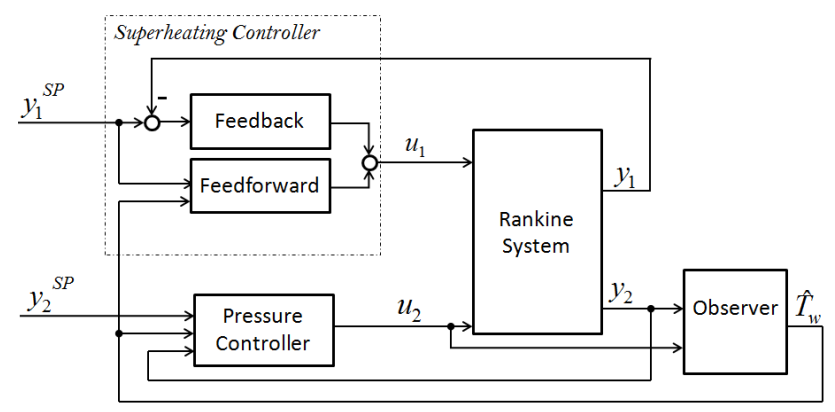

Fig. 3. Proposed closed loop structure for control of $S H$ and $p$. Observer feeds state estimation to the model-based controller.

\section{Control Design}

Let us consider the class of implicit systems of the following form which includes system (6):

$$
\left\{\begin{array}{l}
\dot{x}=F(x, z, u) \\
\Phi(x, z)=0 \\
y=h(x, z)
\end{array}\right.
$$

where $y \in \mathbb{R}^{p}, u \in \mathbb{U} \subset \mathbb{R}^{m},(x, z) \in \mathbb{R}^{n} \times \mathbb{R}^{d}, F, h$ and $\Phi=\left(\Phi_{1}, \ldots, \Phi_{d}\right)^{T}$ are assumed to be sufficiently smooth with respect to their arguments, and

$$
\left.\frac{\partial \Phi}{\partial z}\right|_{(x, z)} \text { is of full rank } \forall(x, z) \in \mathcal{M}
$$

where $\mathcal{M}$ is the set of zeros of $\Phi$ :

$$
\mathcal{M}=\left\{(x, z) \in \mathbb{R}^{n} \times \mathbb{R}^{d} \text {, s.t. } \Phi(x, z)=0\right\} .
$$

Note that this condition guarantees the existence and the uniqueness of the solution $z$ of $\Phi(x, z)=0$, for every $x$.

To transform the implicit system (10) to an Ordinary Differential Equation (ODE) system, we need the following assumption.

Assumption 1: There exists $\epsilon>0$ such that $\frac{\partial \Phi}{\partial z}$ has a full rank on the tubular neighbourhood

$$
\Omega_{\epsilon}=\left\{(x, z) \in \mathbb{R}^{n+d} ;\|\Phi(x, z)\|<\epsilon\right\},
$$

and there exist two functions $\sigma, \widetilde{\sigma}$ of class $\mathcal{K}_{\infty}$ such that:

i) $\forall(x, z) \in \Omega_{\epsilon},\|\Phi(x, z)\| \geq \sigma(\|z-z(x)\|)$

ii) $\forall\left(x^{\prime}, z^{\prime}\right) \in \Omega_{\epsilon}$, we have $\left\|z(x)-z\left(x^{\prime}\right)\right\| \leq \widetilde{\sigma}\left(\left\|z-z^{\prime}\right\|\right)$. From $\mathcal{A}_{1}$ ), we deduce that the following system

$$
\left\{\begin{aligned}
\dot{x} & =F(x, z, u) \\
\dot{z} & =-\left(\left.\frac{\partial \Phi}{\partial z}\right|_{x, z}\right)^{-1}\left(\left.\frac{\partial \Phi}{\partial x}\right|_{x, z}\right) F(x, z, u) \\
y & =h(x, z)
\end{aligned}\right.
$$

is well defined on $\Omega_{\epsilon}$.

Moreover, the trajectories of (10) issued from the manifold $\mathcal{M}$, coincide with those of system (13).

Now, let us use this result to transform our system (6) into an explicit system. To do so, we must verify that Assumption 1 holds. Using the analytic form of $\varphi\left(T_{w}, p\right)$ given by (8), we can show that Assumption 1 is satisfied in the physical domain (for more details on the proof see the appendix).

This done, system (6) can be written as

$$
\left\{\begin{aligned}
\dot{T_{w}} & =f\left(T_{w}, p\right)+u_{2} g\left(T_{w}, t\right) \\
\dot{p} & =-\left(\left.\frac{\partial \varphi}{\partial p}\right|_{T_{w}, p}\right)^{-1}\left(\left.\frac{\partial \varphi}{\partial T_{w}}\right|_{T_{w}, p}\right) \\
& \times\left(f\left(T_{w}, p\right)+u_{2} g\left(T_{w}, t\right)\right),
\end{aligned}\right.
$$

where

$$
\left.\frac{\partial \varphi}{\partial p}\right|_{T_{w}, p}=\beta_{4} \sqrt{\rho(p) p}\left(\begin{array}{l}
\frac{h_{l}(p)-h_{\text {in }}}{\beta_{1}\left(T_{w 1}-T_{1}(p)\right)^{2}} \\
\frac{h_{v}(p)-h_{l}(p)}{\beta_{2}\left(T_{w 2}-T_{2}(p)\right)^{2}} \\
\frac{h_{\text {out }}(p)-h_{v}(p)}{\beta_{3}\left(T_{w 3}-T_{3}(p)\right)^{2}}
\end{array}\right) .
$$

Proposition 1: For every $T_{i}(i=1, \ldots, 3), T_{\text {exh }}$ bounded and for the input $u($.$) which takes values in$ some bounded set $\mathbb{U} \subset \mathbb{R}^{+}$, the solution $T_{w}$ of system

$$
\dot{T}_{w}=f\left(T_{w}, p\right)+u_{2} g\left(T_{w}, t\right), \quad \forall T_{w i}\left(t_{0}\right)=x_{0}
$$

is uniformly bounded with respect to $t$ in $\mathbb{R}^{+}$, i.e. the system (14) is BIBO (bounded-input bounded-output) stable.

Proof: Considering the positive definite quadratic function $V\left(T_{w i}\right)=T_{w i}^{2} / 2$, the derivative of $V$ along the 
trajectories of a dynamical system (16) is given by

$$
\begin{aligned}
\dot{V} & =T_{w i}\left(\beta_{i}\left(T_{i}(t)-T_{w i}\right)+u(t)\left(T_{e x h}(t)-T_{w i}\right)\right) \\
& =-T_{w i}^{2}\left(\beta_{i}+u(t)\right)+T_{w i}\left(\beta_{i} T_{i}(t)+u(t) T_{e x h}(t)\right) \\
& \leq-2 a V+b \sqrt{2 V} \\
& \leq-\sqrt{V}(2 a \sqrt{V}-b \sqrt{2})
\end{aligned}
$$

where $a=\inf \left(\beta_{i}+u(t)\right)$ and $b=$ $\sup \left(\beta_{i} T_{i}(t)+u(t) T_{\text {exh }}(t)\right)$ with $a>0$ and $b \geq 0$. From, (17), we deduce that $\dot{V}$ is negative for $T_{w i}$ such that $\left\{\left|T_{w i}\right| \leq b / a\right\}$. Furthermore, solutions starting from the set $\left\{V\left(T_{w i}\right) \leq(b / a)^{2} / 2\right\}$ at $t_{0}$, remain there for $t>t_{0}$ since $\dot{V}$ is negative on the boundary $V=(b / a)^{2} / 2$.

Based on the same physical conditions used to show that $\left.\frac{\partial \varphi}{\partial p}\right|_{T_{w}, p}<0$, we can state our nonlinear controller in the following proposition.

Proposition 2: Let $k>0$ then, the control ${ }^{1}$

$$
u_{2}\left(T_{w}, p, p^{S P}\right)=\frac{-\varphi_{T_{w}}^{T} f\left(T_{w}, p\right)+k\left(p^{S P}-p\right)}{\varphi_{T_{w}}^{T} g\left(T_{w}, t\right)},
$$

asymptotically stabilizes $p$ to the setpoint $p^{S P}$. Moreover, the input $u_{2}$ and the states of system (6) remain bounded.

Proof: Let $u_{2}\left(T_{w}, p, p^{S P}\right)$ defined as in (18). Since conditions $\mathcal{C}_{1}$ and $\mathcal{C}_{2}$ hold, $u_{2}$ is bounded. Then the second equation of system (14) becomes :

$$
\dot{p}=-\varphi_{p}^{-1} k\left(p^{S P}-p\right) .
$$

Moreover, from proposition 3 , we have $\varphi_{p}<0$. So, obviously, pressure dynamic in (19) is asymptotically attracted by $p^{S P}$.

\section{OBSERVER DESIGN}

To design an observer for the implicit system (10), the authors of [9] gave an implicit observer described by a dynamic system together with an optimization problem permitting to solve the algebraic constraint at each time. Such observer is called an implicit observer.

Definition 1: An implicit observer for system (10) which converges for every input $u \in \mathcal{U}$ is a dynamical system of the form

$$
\left\{\begin{array}{l}
\dot{\hat{x}}=\widehat{F}(\hat{x}, \hat{z}, g, y, u) \\
\dot{g}=G(g, u), \quad g \in O \subset \mathbb{R}^{k} \\
\Phi(\hat{x}, \hat{z})=0,
\end{array}\right.
$$

such that for every $u \in \mathcal{U}$ we have

i) $\forall g(0) \in O$, the trajectory $(g(t))_{t \geq 0}$ lies into a compact subset of $O$.

\footnotetext{
${ }^{1}$ The following notations are used $: \varphi_{p}=\left(\left.\frac{\partial \varphi}{\partial p}\right|_{T_{w}, p}\right), \quad \varphi_{T_{w}}=$ $\left(\left.\frac{\partial \varphi}{\partial T_{w}}\right|_{T_{w}, p}\right)$
}

ii) $\forall r>0 ; \forall(x(0), z(0)) \in \mathcal{M} ; \exists r^{\prime}>0$; $\forall(\hat{x}(0), \hat{z}(0))) \in \mathcal{M},\|(\hat{x}(0)-x(0), \hat{z}(0)-z(0))\|<r^{\prime}$ implies $\|(\hat{x}(t)-x(t), \hat{z}(t)-z(t))\|<r$, for all $t \geq 0$.

iii) $\forall(x(0), z(0)) \in \mathcal{M} ; \quad \forall(\hat{x}(0), \hat{z}(0))) \in \mathcal{M}$, $\lim _{t \rightarrow+\infty}\|(\hat{x}(t)-x(t), \hat{z}(t)-z(t))\|=0$

where conditions $i$ ) and $i i$ ) ensure the stability of the observer, and condition iii) guarantees its convergence. Based on this definition, and using our notations $z=p$ and $x=T_{w}$ with $\left(T_{w}(t), p(t)\right) \in \mathbb{R}^{3} \times \mathbb{R}$ and $y(t)=p$, the gain $G$ is given by a Riccati equation, and an implicit extended Kalman filter (EKF) for system (6) is given by (for more details, see [9], [10])

$\left\{\begin{array}{l}\dot{\hat{T}}_{w}=f\left(\hat{T}_{w}, \hat{p}\right)+u_{2} g\left(\hat{T}_{w}, \hat{p}\right)-S C^{T} R^{-1}(\hat{p}-p) \\ \Phi\left(\hat{T}_{w}, \hat{p}\right)=0 \\ \dot{S}=A S+S A^{T}-S C^{T} R^{-1} C^{T} S+Q \\ S(0)=S(0)^{T}>0,\end{array}\right.$

where $Q$ is a constant symmetric positive definite (SPD) matrix, $R$ is a real positive constant and

$$
A=\left.\frac{\partial\left(f+u_{2} g\right)}{\partial T_{w}}\right|_{\left(\hat{T}_{w}, \hat{p}\right)}, \quad C=\varphi_{p}^{-1} \varphi_{T_{w}}^{T} .
$$

V. Simulation Results

To demonstrate the effectiveness of the control structure described in Fig. 3, this section presents a set of simulation results with realistic operating conditions.

\section{A. Observer validation}

Observer (21) performances in presence of measurement noise can be seen Fig. 4. System simulation is conducted under transient conditions. Observer (21) is initialized with initial condition different from the reference model. The states of the observer are found to converge faster to their target values than the reference model states, if they both start with the same initial conditions (with the same errors). Moreover, it provides an efficient filtering of pressure measurement (Fig. 5) that will be useful to the control feedback.

\section{B. Closed-loop evaluation}

Experimental data for exhaust gas conditions that are representative of a long-haul truck mission, as shown in Fig. 6, were used. Moreover, in order to assess the robustness of the control, errors were considered on two main model parameters: an error of $10 \%$ on the heat exchange coefficient between exhaust gas and evaporator wall, and of $20 \%$ on the wall volume.

Fig. 7 presents estimation results. The states of the reference model that are not measurable and their estimation are compared. Due to the model parameters errors, estimations do not converge exactly to their target values. But they still provide representative values, especially in fast transient periods.

Fig. 8 and 9 validate control capability in regulating the process output $S H$ to its setpoint $S H^{S P}$ despite fast 


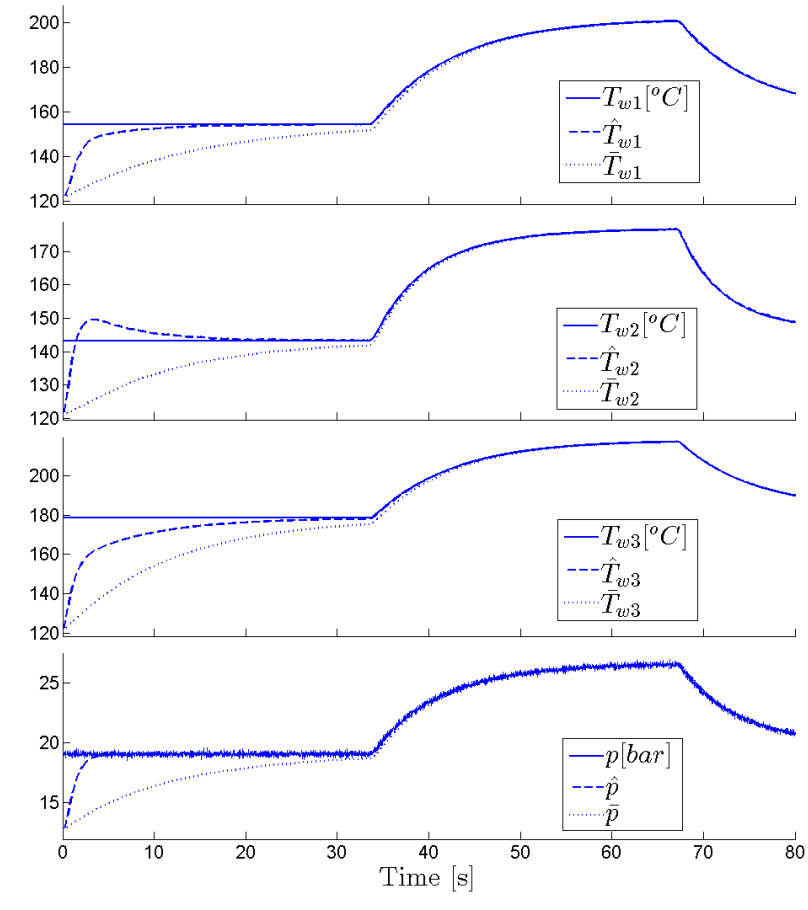

Fig. 4. Simulation results under varying inlets: reference model (solid line), observer (dash line) and reference model with observer initial conditions (dot line).

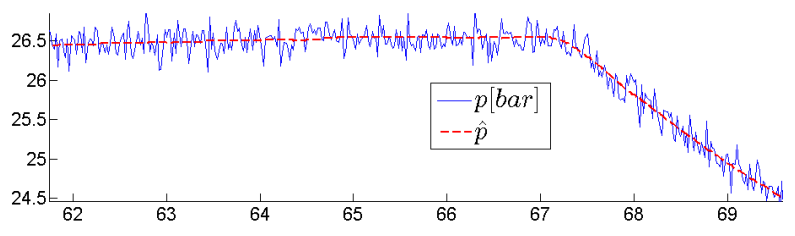

Fig. 5. Zoom on pressure plot of Fig. 4.

transients on exhaust conditions and dramatic variations of pressure. A tight pressure set-point tracking is also demonstrated as long as exhaust gas heat flow is sufficient. Indeed, in the time interval between $1400 \mathrm{~s}$ and $1800 s$, pressure set-point - or equivalently, power production demand - cannot be satisfied due to insufficient exhaust gas heat flow.

Finally, to emphasize benefits of observer, a second simulation is conducted where state estimation is performed via an open-loop model. Fig. 10 compares resulting superheating regulations: it is more than $8 K$ in this case while the maximum error is about $5 K$ when the observer is used. Thus, observer was found to improve robustness of our model-based control strategy.

\section{CONCLUSion}

This paper presents a nonlinear control strategy for an ORC system. The proposed scheme combines a superheat controller, an additional nonlinear controller - allowing pressure set-point tracking or equivalently a tracking of power production demand - and an implicit extended Kalman Filter for wall temperature estimations.

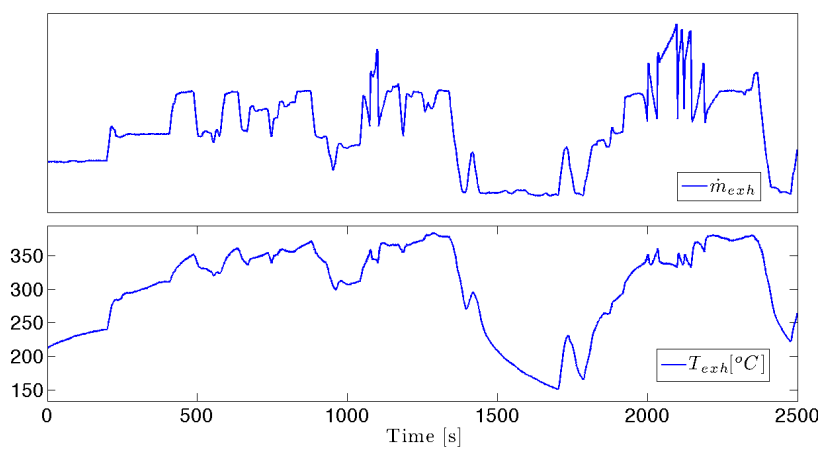

Fig. 6. Experimental data of a truck exhaust gas conditions: mass flow and temperature

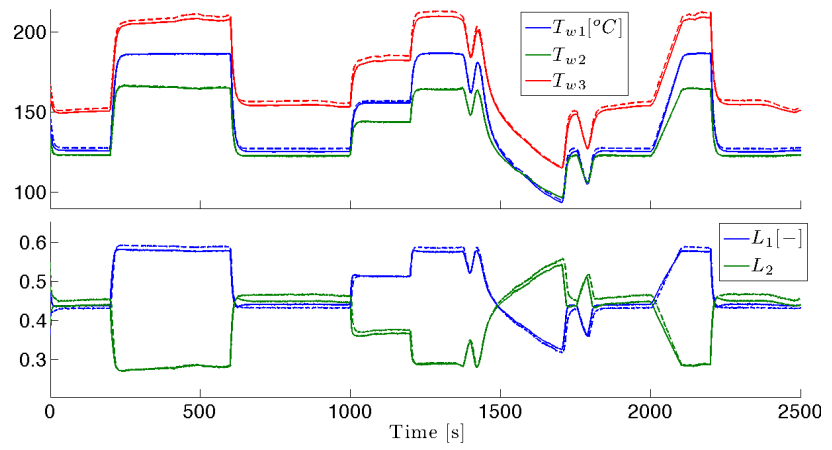

Fig. 7. Estimation (dashed lines) of reference model unmeasurable states (solid lines).

The proposed approach has been illustrated with success in presence of measurement noise, model uncertainties and state initial errors. The experimental implementation of the new control scheme is in progress.

\section{REFERENCES}

[1] C. Sprouse III and C. Depcik. Review of organic rankine cycles for internal combustion engine exhaust waste heat recovery. Applied Thermal Engineering, 51(1-2):711 - 722, 2013.

[2] J. Zhang, W. Zhang, G. Hou, and F. Fang. Dynamic modeling and multivariable control of organic rankine cycles in waste heat utilizing processes. Computers \& Mathematics with Applications, 64(5):908-921, 2012.

[3] J. Zhang, Y. Zhou, S. Gao, and G. Hou. Constrained predictive control based on state space model of organic rankine cycle system for waste heat recovery. In Chinese Control and Decision Conference (CCDC), pages 230 -234, 2012.

[4] G. Hou, R. Sun, G. Hu, and J. Zhang. Supervisory predictive control of evaporator in organic rankine cycle (ORC) system for waste heat recovery. In Advanced Mechatronic Systems (ICAMechS), pages 306-311, 2011.

[5] E. Feru, F. Kupper, C. Rojer, X. Seykens, F. Scappin, F. Willems, J. Smits, B. De Jager, and M. Steinbuch. Experimental validation of a dynamic waste heat recovery system model for control purposes. In SAE World Congress. SAE International, 2013.

[6] T. A. Horst, H. S. Rottengruber, M. Seifert, and J. Ringler. Dynamic heat exchanger model for performance prediction and control system design of automotive waste heat recovery systems. Applied Energy, 105(0):293 - 303, 2013.

[7] J. Peralez, P. Tona, O. Lepreux, A. Sciarretta, L. Voise, P. Dufour, M. Nadri, et al. Improving the control performance of an organic rankine cycle system for waste heat recovery from a heavy-duty diesel engine using a model-based approach. In 


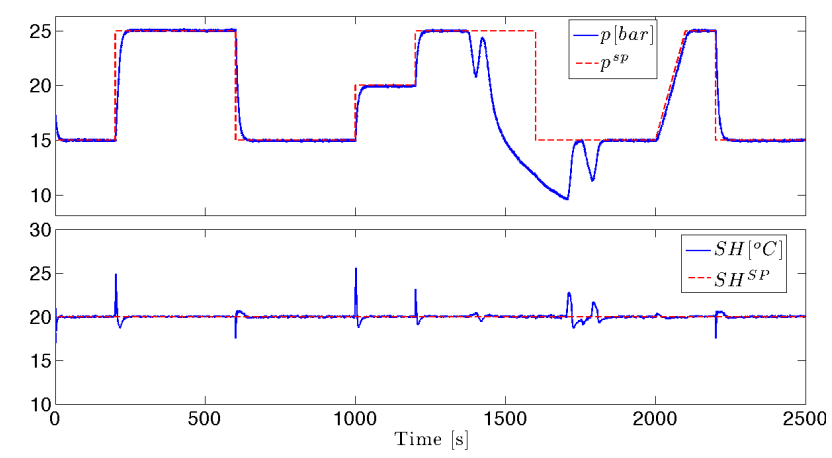

Fig. 8. Pressure set-point $p^{S P}$ tracking and superheating regulation around $S H^{S P}$.

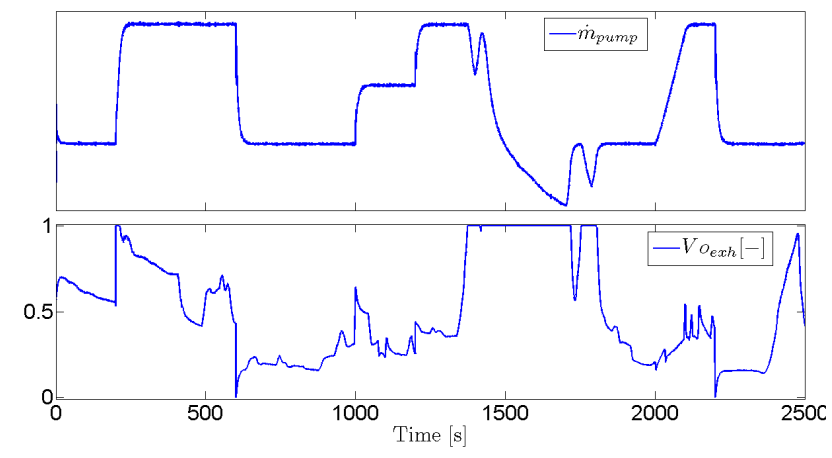

Fig. 9. Evolution of the two control variables: the pump mass flow and evaporator bypass.

2013 IEEE Conference on Decision and Control (CDC), pages 6830-6836, 2013.

[8] J.M. Jensen. Dynamic modeling of thermo-fluid systems with focus on evaporators for refrigeration. PhD thesis, Technical University of Denmark, 2003.

[9] R. Nikoukhah. A new methodology for observer design and implementation. IEEE Transactions on Automatic Control, 43(2):229-234, 1998.

[10] J. Åslund and E. Frisk. An observer for non-linear differentialalgebraic systems. Automatica, 42(6):959-965, 2006.

\section{APPENDIX}

In order to check if this assumption is satisfied, we consider normal operating conditions of an ORC. Namely, the following conditions are considered.

Condition $\mathcal{C}_{1}$ : Pressure $p$ in the evaporator is such that $p<25$ bar and temperature of working fluid $T_{\text {evap, in }}$ at evaporator inlet verifies $10^{\circ} \mathrm{C}<T_{\text {evap, in }}<40^{\circ} \mathrm{C}$.

Condition $\mathcal{C}_{2}: T_{w i}>T_{i}$ for $i=1,2,3$.

Notice that $\mathcal{C}_{1}$ is verified for the considered system and working fluid in normal conditions (greater pressures or inlet temperatures do not match safety conditions and/or efficiency considerations and would lead to system shutdown by the control system supervisor). $\mathcal{C}_{2}$ means that heat is transferred from wall to fluid which might not be verified only during start-up operation. However, in practice, none of these conditions is restrictive.

Proposition 3: Since conditions $\mathcal{C}_{1}, \mathcal{C}_{2}$ hold, we get $\left.\frac{\partial \Phi}{\partial p}\right|_{\left(T_{w}, p\right)}<0$.

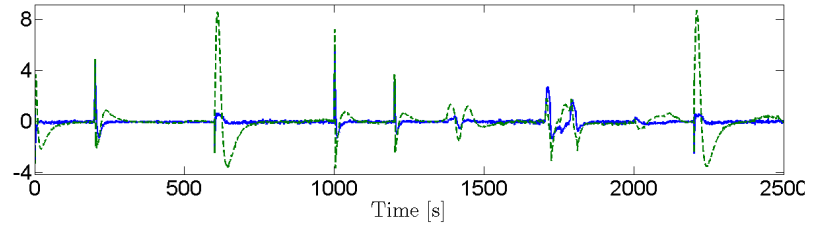

Fig. 10. Superheating regulation error $\left(S H-S H^{S P}\right)$ with observer (solid blue line) and with state estimation performed by an openloop model (dash green line).

To proof this proposition let us show the following sufficient conditions:

1) $\left.\left.\left.\frac{\partial L_{3}}{\partial p}\right|_{\left(T_{w}, p\right)}>0, \quad 2\right)\left.\frac{\partial L_{2}}{\partial p}\right|_{\left(T_{w}, p\right)}>0, \quad 3\right)\left.\frac{\partial L_{1}}{\partial p}\right|_{\left(T_{w}, p\right)}>0$, where $L_{i}$ are given by (9).

1) Let $L_{3}=\frac{N_{3}}{D_{3}}$, where $N_{3}=u_{1}\left(h_{\text {out }}(p)-h_{v}(p)\right)$ and $D_{3}=\beta_{3}\left(T_{w 3}-T_{3}(p)\right)$, according to (8). Then $\operatorname{sign}\left(\left.\frac{\partial L_{3}}{\partial p}\right|_{(.)}\right)=\operatorname{sign}\left(\left.\frac{\partial N_{3}}{\partial p}\right|_{(.)} D_{3}-\left.N_{3} \frac{\partial D_{3}}{\partial p}\right|_{(.)}\right)$.

Clearly $N_{3}>0$ and $\left.\frac{\partial D_{3}}{\partial p}\right|_{(.)}<0$ (the evaporation temperature $T_{2}$ increases with $p$ ). Moreover $D_{3}>0$ (condition $\mathcal{C}_{1}$ ). Fig. 11(a) shows that $\left.\frac{\partial N_{3}}{\partial p}\right|_{(.)}>0$ for the considered pressure range. So $\left.\frac{\partial L_{3}}{\partial p}\right|_{(.)}>0$.

2) Similar to 1), let $L_{2}=\frac{N_{2}}{D_{2}}$, and notice that $N_{2}>0$, $\left.\frac{\partial D_{2}}{\partial p}\right|_{(.)}<0$ and $D_{2}>0$. Figure 11(a) shows that $\left.\frac{\partial N_{2}}{\partial p}\right|_{(.)}>0$. So $\left.\frac{\partial L_{2}}{\partial p}\right|_{(.)}>0$.

3) Similar to 1), let $L_{1}=\frac{N_{1}}{D_{1}}$, and notice that $N_{1}>$ $0,\left.\frac{\partial D_{1}}{\partial p}\right|_{(.)}<0$ and $D_{1}>0$. Figure 11(b) shows that $\left.\frac{\partial N_{1}}{\partial p}\right|_{(.)}>0$ for the considered pressure and disturbance range. So $\left.\frac{\partial L_{2}}{\partial p}\right|_{(.)}>0$.

Thus, $\varphi\left(T_{w}, p\right)$ decreases monotonically with $p$. Consequently, $\left.\frac{\partial \Phi}{\partial p}\right|_{\left(T_{w}, p\right)}$ has a full rank in the physical domain.
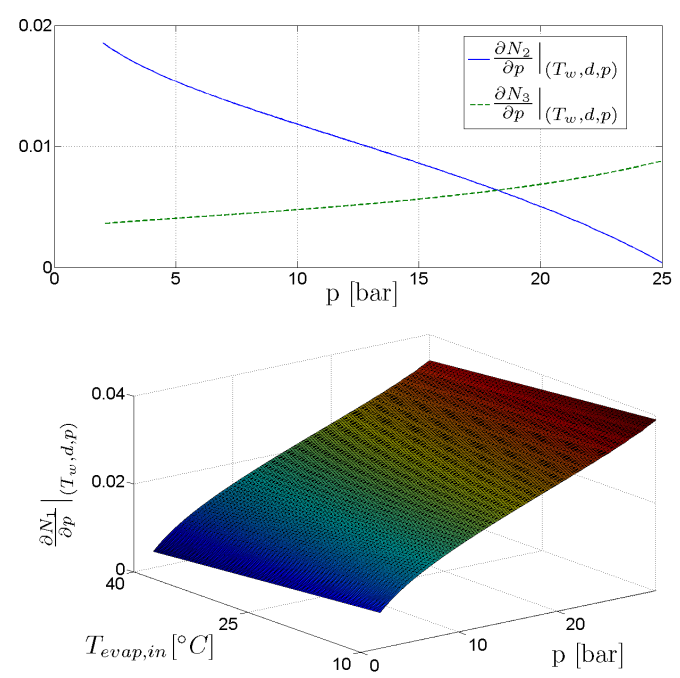

Fig. 11. $\quad N_{1}$ (b: bottom), $N_{2}$ (a: top) and $N_{3}$ (a: top) increase with pressure (here $S H^{S P}$ equal $30^{\circ} \mathrm{C}$ ). 